\title{
Metabolic acidosis and benign intracranial hypertension in infants: an adverse drug reaction
}

\author{
P M G Punchihewa ${ }^{1}$, M K S Gunawardena ${ }^{2}$ \\ Sri Lanka Journal of Child Health, 2003; 32: 20-2
}

(Key words: metabolic acidosis, benign intracranial hypertension, infant)

Nalidixic acid is a 4-quinolone antimicrobial agent used in the treatment of uncomplicated lower urinary tract infection and shigellosis. The recommended dose for a child is $50-55 \mathrm{mg} / \mathrm{kg}$ body weight daily in 4 divided doses. The drug is rapidly and almost completely absorbed from the gastrointestinal tract (GIT) and the plasma half-life is about 2.5 hours. Nalidixic acid and its metabolites are excreted rapidly in the urine. This drug should ideally be avoided in children younger than three months of age.

About $8 \%$ of patients experience adverse effects with nalidixic acid, most frequently involving the GIT, skin and the central nervous system (CNS)1. Benign intracranial hypertension $(\mathrm{BIH})$, although known to occur at any age, is commoner in infants and young children $^{2,3,4}$. Severe metabolic acidosis has been reported in infants following a single therapeutic dose and in adults following an overdose of the drug ${ }^{1}$. Haemolytic anaemia and thrombocytopenia, although less well known, have been reported ${ }^{1,5}$. We report two cases of misuse of nalidixic acid which resulted in $\mathrm{BIH}$ and severe metabolic acidosis.

\section{Case 1}

A 5-day-old baby boy was admitted with a history of excessive crying and poor sucking for 1 day. He had been treated for "loose stools" with syrup nalidixic acid for 24 hours prior to the onset of symptoms. Apart from the history of "loose stools" the baby has been perfectly well. Soon after admission, he developed focal clonic convulsions. The baby has had an uncomplicated vaginal delivery, to non-consanguineous parents, with a birth weight of $2.8 \mathrm{~kg}$, and had been exclusively breastfed.

On examination, the baby was ill and irritable and weighed $2.8 \mathrm{~kg}$. He was tachypnoeic with a respiratory rate of $80 / \mathrm{min}$. His hydration and the peripheral ${ }^{1}$ Consultant Paediatrician, ${ }^{2}$ Paediatric
Teaching Hospital, Karapitiya.

(Received on 19 April 2002) circulation were good. The anterior fontanelle was tense and bulging. There was no papilloedema and no cranial nerve palsies. The lungs were clear. Funduscopy was normal.

The initial arterial blood gas analysis revealed severe metabolic acidosis with a $\mathrm{pH}$ of 7.08 and a base deficit of $25.8 \mathrm{meq} / 1$. The random blood sugar and serum electrolytes, including serum calcium, were normal. The cerebrospinal fluid (CSF) analysis was normal and the blood culture remained sterile.

Ultrasound scan of the brain revealed a normal ventricular system with no midline shift.

Our initial differential diagnoses were septicaemia, $\mathrm{BIH}$ and metabolic acidosis secondary to nalidixic acid or an inborn error of metabolism.

The convulsions were controlled with intravenous phenobarbitone. Fluids were restricted and a single dose of $20 \%$ mannitol was given to reduce the cerebral oedema. Intravenous crystalline penicillin and cefotaxime were commenced as for meningitis/septicaemia and the drugs were discontinued once the blood culture and CSF reports were available. The metabolic acidosis was corrected with repeated slow infusions of sodium bicarbonate. This baby was managed in the neonatal intensive care unit for forty eight hours and was discharged from hospital on the ninth day after admission.

Visual evoked potentials (VEP) done at 4 weeks of age did not reveal any visual loss.

\section{Case 2}

A4-month-old adopted baby with Down syndrome, ventricular septal defect and surgically corrected bilateral cataract was admitted with a history of mucoid diarrhoea of 2 days duration and poor sucking, He had been treated with a drug, by a general practitioner, which was later identified as 
nalidixic acid in a dose of $125 \mathrm{mg} 6$ hourly. He did not have convulsions.

On admission the baby weighed $3.5 \mathrm{~kg}$. His hydration and peripheral circulation were satisfactory. He was tachypnoeic with a respiratory rate of 60 per min. The anterior fontanelle was tense and bulging but the baby remained afebrile. Fundoscopy revealed bilateral papilloedema. Though the heart rate was $146 / \mathrm{min}$. there was no hepatomegaly and the lungs were clear.

Our initial diagnosis was septicaemia /meningitis or a metabolic disorder. BIH and metabolic acidosis due to nalidixic acid, was considered after the drug was identified as nalidixic acid.

The initial arterial blood gas analysis revealed a severe metabolic acidosis with a $\mathrm{pH}$ of 7.18 and a base deficit of $22.4 \mathrm{mEq} / 1$. The white cell count showed a neutrophil leucocytosis. Blood culture was sterile. CSF analysis was done after the papilloedema resolved and was found to be normal. Ultrasound scan of the brain showed normal ventricular system with no midline shift. The baby was initially resuscitated with normal saline $20 \mathrm{ml} / \mathrm{kg}$ boluses. Correction of metabolic acidosis needed several slow intravenous infusions of sodium bicarbonate.

Treatment for septicaemia/meningitis was discontinued once the reports were available. The baby was discharged one week after admission. The Flash VEP was repeatedly absent when visual evoked potential was performed after a week

\section{Discussion}

Two babies, one only 5 days old and the other 4 months, had received almost 3 times the therapeutic dose of nalidixic acid and developed metabolic acidosis and benign intracranial hypertension. The clinical manifestations of metabolic acidosis are often nonspecific. The most important physical sign is hyperventilation, the extreme of which is the deep, rapid respiration needed for respiratory compensation. Severe acidosis can cause decrease in peripheral vascular resistance and cardiac ventricular function resulting in hypotension, pulmonary oedema and tissue hypoxia.

Metabolic acidosis cannot be confirmed by arterial blood gas analysis in most local hospitals. Tachypnoea could be mistakenly attributed to a respiratory or cardiac cause. If detected late, metabolic acidosis may not only prolong the period of morbidity, but may even result in death.
The clinical features of benign intracranial hypertension, too, are non-specific, and in an infant or neonate, the only clinical features may be excessive crying, refusal of feeds, vomiting and a bulging tense anterior fontanelle. Papilloedema may be present. Seizures are described rarely and should be attributed to BIH only after excluding other causes of a seizure ${ }^{1,6}$.

$\mathrm{BIH}$ has been reported with drugs, such as tetracycline, nitrofurantoin, steroids, hypervitaminosis A, and ciprofloxacin ${ }^{7,8,9}$. Metabolic acidosis has been reported in the literature ${ }^{10}$.

Both babies had refusal of feeds with excessive crying and on examination the anterior fontanelle was found to be bulging. One baby developed focal clonic convulsions and the other had papilloedema. Cerebrospinal fluid analysis in both babies excluded meningitis. Ultrasound scan of the brain revealed normal sized ventricles which is a prerequisite for the diagnosis of BIH.

Loss of visual function is the only serious permanent complication of BIH. Visual field loss or decreased visual acuity in children has been reported in 13$27 \%{ }^{6}$. The most sensitive indicator is visual field testing which cannot be used in younger children, especially under 7 years. VEP is considered to be an insensitive indicator of early visual loss and changes are infrequent and occur only with severe loss of vision. But our two patients, one an infant and the other a neonate could only have VEP done to assess their vision as none of the other indicators of visual function could be applied to these babies. The baby who had repeatedly absent flash VEP most likely had optic nerve damage following $\mathrm{BIH}$.

Our management included the immediate withdrawal of nalidixic acid and the reduction of cerebral oedema. We did not have to use the other forms of medical management available, including therapy with acetazolamide, steroid or frusemide and repeated lumbar punctures. Surgical management in the form of lumbar peritoneal shunting and optic nerve sheath fenestration can be done in the presence of deteriorating vision ${ }^{6}$.

Although haematological effects have been described as adverse effects of nalidixic acid, these two babies had normal haematological indices.

We have reported these two cases to the Adverse Drug Reaction section of the Drug Evaluation sub committee for necessary action. We have also 
educated the two medical officers who had wrongly prescribed the nalidixic acid.

\section{Conclusions}

Nalidixic acid, which is an effective drug in the treatment of lower urinary tract infections and shigellosis, is indiscriminately and inappropriately used even by MBBS qualified doctors. It is vital to carry out an awareness campaign regarding its use and misuse. Metabolic acidosis is a lesser known adverse effect of nalidixic acid and should be borne in mind in the case of an unexplained metabolic acidosis. Drugs prescribed in wrong doses by qualified doctors have been reported not infrequently. It is vital that dosage should be checked when prescribing, especially in children.

\section{References}

1. Reynold J E S (Editor). Martin Dale - The extra pharmacopoeia. 29th ed. London; Pharmaceutical Press, 1989.

2. Boreus L O, Sundstrom B. Intracranial hypertension in a child with nalidixic acid. British Medical Journal 1967; 2: 744-5.

3. Mukherjee A, Dutta P, Lahiri M, Singha S, et al. Benign intracranial hypertension after nalidixic acid overdose in infants (Letter). The Lancet 1990; 335:1602.
4. Deanna T, Guignard J P. Acute intracranial hypertension after nalidixic acid administration. Archives of Diseases in Childhood 1974; 49(9): 743.

5. Belton M, Vaughan Jones R. Haemolytic anaemia due to nalidixic acid (Letter). The Lancet 1965; 2: 691 .

6. Soler S, Cox T, Calver D M, Robinson R O. Diagnosis and management of benign intracranial hypertension. Archives of Diseases in Childhood 1998; 78: 89-94.

7. Lucas $\mathrm{G}$ N. Benign intracranial hypertension in infancy (The "bulging fontanelle" syndrome). Ceylon Journal of Child Health 1986; 15: 25-7.

8. Winrow A P, Supramaniam G. Benign intracranial hypertension after ciprofloxacin administration. Archives of Diseases in Childhood 1990; 65:1165-6.

9. Pearson M G, Littlewood S M, Bowden A N. Tetracycline and benign intracranial hypertension. British Medical Journal 1981; 282: 568-569.

10. Dash H, Mills J. Severe Metabolic Acidosis with Nalidixic Acid Overdose. Annals of Internal Medicine 1976; 84(5): 570-1. 
